\title{
Increasing the clinical efficacy of NK and antibody-mediated cancer immunotherapy: potential predictors of successful clinical outcome based on observations in high-risk neuroblastoma
}

\author{
Tony A. Koehnn ${ }^{1+}$, Lori L. Trimble ${ }^{1+}{ }^{\text {, Kory L. Alderson }}{ }^{1}$, Amy K. Erbe ${ }^{1}$, Kimberly A. McDowell ${ }^{1}$, Bartosz \\ Grzywacz' ${ }^{2}$, Jacquelyn A. Hank ${ }^{1,3}$ and Paul M. Sondel 1,3,4 *
}

${ }^{1}$ Department of Human Oncology, University of Wisconsin Madison, Madison, WI, USA

${ }^{2}$ Department of Pathology, University of Wisconsin Madison, Madison, WI, USA

${ }^{3}$ Paul P. Carbone Comprehensive Cancer Center, University of Wisconsin Madison, Madison, WI, USA

${ }^{4}$ Department of Pediatrics, University of Wisconsin Madison, Madison, WI, USA

\section{Edited by:}

Alexandre Arcaro, University of Bern Switzerland

Reviewed by:

Todd Skaar, Indiana University Medical Center, USA

Francois M. Vallette, Institut National de la Santé et de la Recherche

Médicale, France

*Correspondence:

Paul M. Sondel, Paul P. Carbone Comprehensive Cancer Center,

University of Wisconsin Madison, 4159 MACC Fund UW Childhood

Cancer Research Wing, WIMR, 1111

Highland Avenue, Madison, WI

53705-2275, USA.

e-mail:pmsondel@humonc.wisc.edu

${ }^{+}$Co-first authors.
Disease recurrence is frequent in high-risk neuroblastoma (NBL) patients even after multimodality aggressive treatment [a combination of chemotherapy, surgical resection, local radiation therapy, autologous stem cell transplantation, and cis-retinoic acid (CRA)]. Recent clinical studies have explored the use of monoclonal antibodies (mAbs) that bind to disialoganglioside $\left(\mathrm{GD}_{2}\right)$, highly expressed in $\mathrm{NBL}$, as a means to enable immune effector cells to destroy NBL cells via antibody-dependent cell-mediated cytotoxicity (ADCC). Preclinical data indicate that ADCC can be more effective when appropriate effector cells are activated by cytokines. Clinical studies have pursued this by administering anti-GD $2 \mathrm{mAb}$ in combination with ADCC-enhancing cytokines (IL2 and GM-CSF), a regimen that has demonstrated improved cancer-free survival. More recently, early clinical studies have used a fusion protein that consists of the anti-GD $\mathrm{GA}_{2} \mathrm{mAb}$ directly linked to IL2, and anti-tumor responses were seen in the Phase II setting. Analyses of genes that code for receptors that influence ADCC activity and natural killer (NK) cell function [Fc receptor ( $F c R)$, killer immunoglublinlike receptor (KIR), and KIR-ligand (KIR-L)] suggest patients with anti-tumor activity are more likely to have certain genotype profiles. Further analyses will need to be conducted to determine whether these genotypes can be used as predictive markers for favorable therapeutic outcome. In this review, we discuss factors that affect response to mAb-based tumor therapies such as hu14.18-IL2. Many of our observations have been made in the context of NBL; however, we will also include some observations made with mAbs targeting other tumor types that are consistent with results in NBL. Therefore, we hypothesize that the NBL observations discussed here may also be relevant to mAb therapy for other cancers, in which ADCC is known to play a role.

Keywords: ADCC, KIR, FcR, neuroblastoma, immunocytokine, mAb, IL2

\section{INTRODUCTION}

Neuroblastoma is the most common extracranial solid tumor in children, accounting for $8-10 \%$ of all childhood cancers with approximately 600 new cases diagnosed in the United States each year (Navid et al., 2009). Greater than $60 \%$ of patients older than 1 year of age have metastatic tumors (Modak and Cheung, 2007), and for these high-risk patients, the prognosis is often poor. Even with the aggressive multi-modal treatment
Abbreviations: ADCC, antibody-dependent cell-mediated cytotoxicity; AIS, activating immune synapse; AML, acute myeloid leukemia; ALL, acute lymphoid leukemia; ASCT, aAutologous stem cell transplant; CD16, FcR $\gamma$ IIIa; CD20, Blymphocyte antigen expressed on all mature B-cells; CD32, FcR $\gamma \mathrm{II}$; ch14.18, chimeric 14.18 anti-GD $-\mathrm{GD}_{2} \mathrm{mAb}$; COG, Children's Oncology Group; CRA, cis-retinoic acid; CRC, colorectal cancer; EGFR, epidermal growth factor receptor; F, phenylalanine; FcR, receptor for $\mathrm{Fc}$ region of immunoglobulin; $\mathrm{GD}_{2}$, disialoganglioside; GM-CSF, granulocyte macrophage colony stimulating factor; H, histidine; HER2, human epidermal growth factor receptor-2; HLA, human leukocyte antigen; HSCT, hematopoietic stem cell transplantation; hu14.18, humanized 14.18 anti-GD 2 mAb; hu14.18-IL2, humanized 14.18-IL2 IC; IC, immunocytokine; IFN- $\gamma$, interferon$\gamma ;$ IL2, interleukin 2; IL2R, IL2 receptor; KIR, killer immunoglobulin-like receptor;
KIR-L, killer immunoglobulin-like receptor ligand; K-ras, Kirsten rat sarcoma oncogene homolog; $\mathrm{mAb}$, monoclonal antibody; MHC-I, major histocompatibility complex class I; MIP-1 $\alpha$, macrophage inflammatory protein- $1 \alpha$; MRD, minimal residual disease; NCRs, natural cytotoxicity receptors; NBL, neuroblastoma; NK, natural killer; NKG2A/CD94, inhibitory NK cell receptor, a dimer of NKG2A and CD94; NKG2D, activating NK cell receptor; NKL, NK-like cell line expressing little or no CD16; PCR-SSP, PCR amplification with sequence-specific primers; PCR-SSOP, PCR amplification using a sequence-specific oligonucleotide probe; $\mathrm{R}$, arginine; RANTES, regulated-upon activation, normal $\mathrm{T}$ expressed cytokine; SBT, sequence-based typing; SNP, single nucleotide polymorphism; TNF- $\alpha$, tumor necrosis factor- $\alpha ; \mathrm{V}$, valine. 
of surgery, chemotherapy, radiotherapy, CRA, and autologous stem cell transplantation (ASCT), the recent (as of 2010) 3-year event-free survival remained as low as $30 \%$, often due to recurrence caused by refractory minimal residual disease (MRD; Yang and Sondel, 2010). More effective treatment strategies are highly sought, including advances via immunotherapeutic options (Yu et al., 2010), currently being further developed in clinical trials.

Neuroblastoma is a tumor of neuroectodermal origin and overexpresses $\mathrm{GD}_{2}$. Since $\mathrm{GD}_{2}$ expression is restricted to few normal tissue types, such as peripheral nerves, and its normal level of expression is relatively low, it is a viable target for tumorselective immunotherapy involving anti- $\mathrm{GD}_{2}$ monoclonal antibodies (mAbs) and their derivatives. Anti-GD $\mathrm{GAb}_{2} \mathrm{mav}$ have evolved from fully murine (3F8 and 14.G2a) to chimeric (ch14.18) to humanized (hu14.18) reagents. One recent preclinical development in anti-GD $\mathrm{G}_{2}$ immunotherapy is an antibody that recognizes the $\mathrm{O}$-acetyl derivative of GD2 (OAcGD2; Alvarez-Rueda et al., 2011). Preclinical and clinical testing is also proceeding with hu14.18-IL2, an immunocytokine (IC) comprised of a humanized form of the anti-GD $\mathrm{GAb}_{2}$ genetically linked to two molecules of IL2 (Gillies et al., 1992; Lode et al., 1998a;Shusterman et al., 2010). These immunotherapies exploit the cytolytic activities of NK cells via CD16, the FcR found on NK cells, which can cause antibodydependent cell-mediated cytotoxicity (ADCC) upon binding to the Fc portion of such mAbs or ICs (Weiner et al., 2009). The degree of cytotoxicity is dependent upon a number of factors including, but not limited to: the activation state of NK cells, the affinity of CD16, and inhibitory signaling via KIRs (Becknell and Caligiuri, 2008). This manuscript reviews recent findings in clinical and preclinical trials related to each of these factors and provides a hypothesis regarding the potential for genetic screening of patients for favorable KIR/KIR-L and FcR genotypes for immunotherapeutic treatment of NBL in the context of NK-based and mAb-mediated cancer immunotherapy. This neuroblastoma (NBL)-based hypothesis may potentially apply to other cancers for which mAb-mediated treatment has been shown to involve ADCC.

\section{NATURAL KILLER CELLS AND ANTIBODY-DEPENDENT CELL-MEDIATED CYTOTOXICITY}

Natural killer cells are large granular lymphocytes of the innate immune system responsible for elimination of infected and transformed cells as well as cytokine production. NK cells recognize and destroy target cells via immune synapses formed through surface-expressed receptors on the NK cells and corresponding ligands on target cells (Orange, 2008). NK cell-mediated cytotoxicity is directed toward specific target cells, based on the balance of recognition of appropriate membrane structures by activating and inhibitory receptors expressed on the NK cell (Leung, 2011). The mechanisms of destruction are multi-modal: the release of cytotoxic granules such as granzymes and perforin and the expression of Fas ligand (Fas-L) and TNF-related apoptosis inducing ligand (TRAIL) are mechanisms used by NK cells to kill target cells (Zompi and Colucci, 2005). NK cells can further shape the immune response by secreting cytokines (such as IFN- $\gamma$, TNF$\alpha$, and GM-CSF), and chemokines (for example MIP- $1 \alpha$ and RANTES; Oliva et al., 1998; Biron et al., 1999). The level of NK response is ultimately determined by the balance of signals from activating and inhibitory receptors (Moretta et al., 2005). NK cells express a variety of activating (NCRs, NKG2D, KIRs, and CD16) and inhibitory receptors (NKG2A/CD94 and KIRs; Farag and Caligiuri, 2006). Also important for the effector-target interaction are adhesion molecules (CD2 and LFA-1) and cytokine receptors (IL2R) that promote adhesion and activate NK cells (Orange et al., 2003; Gubbels et al., 2011).

Antibody-dependent cell-mediated cytotoxicity is an important anti-tumor mechanism of the innate immune system, mediated by leukocytes with FcRs (NK cells, monocytes, macrophages, and neutrophils). When a tumor is treated with a tumor-specific $\mathrm{mAb}$, the variable regions of the Fab portions of the mAbs bind to the antigens on the tumor. The Fc regions of the tumor-bound $\mathrm{mAbs}$ project from the tumor cell surface allowing interactions with effector cells that express FcRs. The binding of many mAb molecules to the surface of a tumor cell creates a lattice of exposed Fc regions allowing for multiple Fc-FcR interactions. When multiple CD16 molecules on an effector cell (i.e., NK cell) are engaged simultaneously, immunoreceptor tyrosine-based activation motif (ITAM)-mediated downstream signaling events occur that lead to triggering of cytotoxic mechanisms and the destruction of mAb-coated tumor cells (Ravetch and Bolland, 2001; Farag et al., 2003).

Leukocytes express a variety of receptors from the FcR family. The most important activating FcRs for ADCC include CD16 (Fc $\gamma$ RIIIa; expressed primarily on NK cells) and CD32 (Fc $\gamma$ RIIa; expressed on monocytes, macrophages, and neutrophils; Nimmerjahn and Ravetch, 2008). CD16 and CD32 bind to the Fc portion of human immunoglobulin molecules with the highest affinity for the IgG1 isotype (Jefferis, 2007); thus human mAbs of the IgG1 isotype induce a more robust ADCC response compared to other heavy chain isotypes. For this reason, most therapeutic mAbs approved for clinical application are of the human IgG1 isotype (Schrama et al., 2006).

The importance of FcRs in mAb-based cancer therapy has been previously demonstrated with the application of multiple tumorspecific mAbs in both preclinical and clinical settings. Using mice lacking FcRs, Clynes et al. (2000) showed that the anti-tumor activity of therapeutic mAbs was diminished in Fc $\gamma \mathrm{R}$ knockout mice compared to wild-type mice, indicating the importance of FcRs in tumor cell clearance. Studies have demonstrated the importance of ADCC as a tumor clearing mechanism in mAb therapies (Basham et al., 1988; Primus et al., 1994). Many therapeutic mAbs currently used clinically to treat cancer, such as Rituximab (antiCD20), Trastuzumab (anti-HER2), Cetuximab (anti-EGFR), and ch14.18 (anti-GD 2 ), likely utilize ADCC as an anti-tumor mechanism, although it is difficult to determine the necessity for NK cell-mediated ADCC in humans (Adams and Weiner, 2005).

\section{CYTOKINE ACTIVATION OF NK CELLS AND HU14.18-IL2 IC}

Several preclinical studies had shown that IL2, a potent activator of NK cells, augments NK-mediated ADCC when used to treat NK cells in vitro, and in vivo administration of IL2 increased the ability of circulating NK cells from cancer patients to mediate ADCC (Hank et al., 1988, 1990, 1993; Sosman et al., 1990). A recent phase III NBL study conducted by the Children's Oncology Group 
(COG) combined mAb treatment with cytokine administration (Yu et al., 2010). Patients that received then-standard therapy of 13-cis retinoic acid (CRA) along with an immunotherapy regimen of ch14.18 mAb, IL2, and GM-CSF showed improved response as compared to the control group that received only CRA. There was a significant improvement in 2-year overall survival (86 vs. $75 \%$; $p=0.02$ ) and 2 -year event-free survival (66 vs. $46 \% ; p=0.01$ ) in children with MRD who received the immunotherapy regimen (Yu et al., 2010). Of note, a study previously conducted in Germany of ch14.18 mAb treatment of children with NBL initially found no therapeutic advantage to treatment with the ch14.18 mAb alone (Simon et al., 2004). A long term follow-up of the German study did demonstrate an advantage in survival, but not in event-free survival (considered to be a more direct indicator of anti-tumor effect of the therapy), for those children that received the ch14.18 (Simon et al., 2011). Importantly, this German study employed ch14.18 alone, without the administration of IL2 and GM-CSF, which suggests that the clinical benefit realized in the COG study was due, at least in part, to the activation of innate immune cells by the addition of IL2 and GM-CSF.

To further advance the therapeutic benefits of this combinatorial therapy, hu14.18-IL2 IC was developed from the wellcharacterized ch14.18-IL2 IC (Yamane et al., 2009). Ch14.18-IL2 was constructed by fusing the gene sequence for human IL2 to the human $C \gamma 1$ gene at the carboxy-terminus of each IgG1 heavy chain of ch14.18 mAb (Gillies et al., 1992). This genetic linking enables the delivery of IL2 to the tumor microenvironment (Lode et al., 1998a; Johnson et al., 2007). In contrast to ch14.18-IL2, the antibody portion of hu14.18-IL2 has been $98 \%$ humanized to minimize anti-mouse antibody responses (Yamane et al., 2009). Hu14.18-IL2 IC is a next generation anti-GD GDer- $_{2}$ apeutic agent that holds promise in NBL treatment. In mice, hu14.18-IL2 IC exhibits superior anti-tumor effects compared to simultaneous administration of similar amounts of hu14.18 mAb and IL2 administered as separate agents (Lode et al., 1998b; Gillies et al., 2005). The anti-tumor effect is primarily NK cell-mediated (Lode et al., 1998b) and more pronounced in mice with nonbulky disease (Neal et al., 2004b). As demonstrated in a murine NBL study (Neal et al., 2004a), increased Major Histocompatibility Complex Class I (H-2 class I, the mouse equivalent of HLA-I in humans, KIR-L) expression appears to be a mechanism by which tumor cells can escape hu14.18-IL2 IC-induced, NK-mediated, immunotherapy. A similar escape mechanism has been shown in B cell-lymphoma upon Rituximab treatment (Borgerding et al., 2010), suggesting that ligation of inhibitory receptors (KIRs) on NK cells by MHC class I (KIR-L) on tumor cells can inhibit ADCC responses.

The superior anti-tumor effects of hu14.18-IL2 IC (over the combination of hu14.18 mAb and soluble IL2 administered simultaneously) may be due, in part, to contributions from IL2Rs that are able to interact with IL2 molecules uniquely presented on the surface of 14.18-IL2 IC-treated tumor cells. Gubbels et al. (2011) used an NK cell line (NKL) that expresses little or no CD16, to demonstrate that hu14.18-IL2 IC can facilitate NK cell conjugation (in vitro) to tumor cells via the NK cells' IL2Rs (Figure 1). Also, interaction with hu14.18-IL2 IC-coated tumor cells via the NK cells' IL2Rs causes the polarization of NK cell adhesion and effector molecules, resulting in the formation of an activating immune synapse (AIS) necessary for the subsequent destruction of tumor cells (Gubbels et al., 2011). Thus the improved anti-tumor efficacy

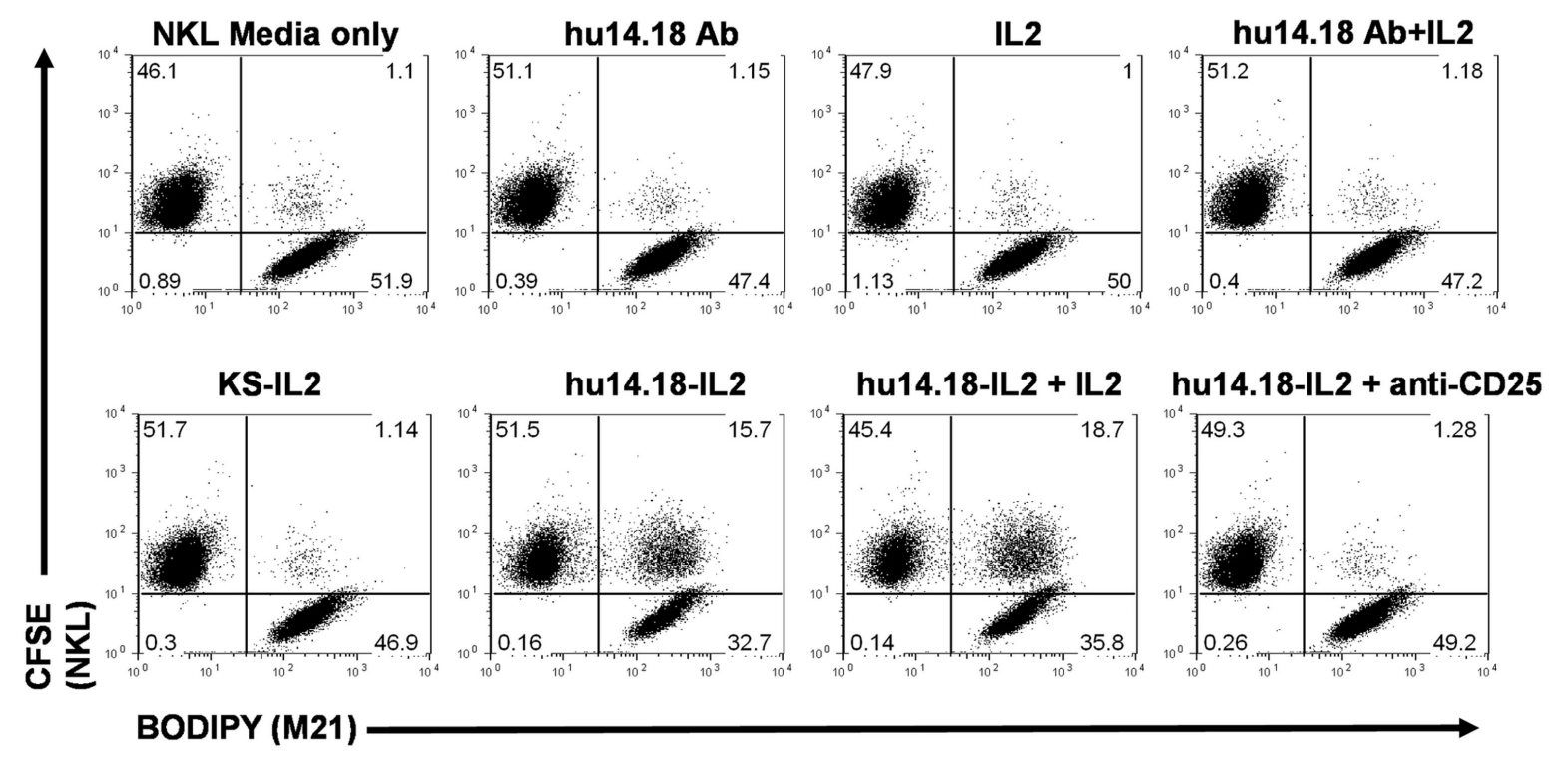

FIGURE 1 | 14.18-IL2 IC increases conjugate formation between M21 $\left(\mathrm{GD}_{2}{ }^{+}\right.$tumor cells) and NKL cells, and conjugate formation can be blocked with an anti-CD25 (IL2R $\alpha$-chain) mAb in flow cytometry conjugate assays. M21 and NKL cells were dyed with BODIPY and CFSE respectively, incubated together with the depicted treatments and analyzed for conjugate formation. huKS-IL2 IC, which recognizes EpCAM not GD 2 , was added to demonstrate specificity of the hu14.18-IL2 IC. Pre-treatment with blocking anti-CD25 mAb almost completely abrogates conjugate formation. Numbers in dot plots indicate percentage of total counted events in each quadrant, with the upper right quadrant indicating 2-color conjugate events. The results are representative of three independent experiments (Reproduced here, with permission, from Gubbels et al., 2011). 
of hu14.18-IL2 IC may be attributed, in part, to its ability to facilitate the formation of immune synapses via the IL2R. IL2 activation of NK cells can increase the expression of activating receptors on the NK cell thereby tipping the balance in favor of activation and reducing the inhibition mediated by KIR/KIR-L interactions (Huenecke et al., 2010). Taken together, these data imply that NK cells might use the hu14.18-IL2 IC to mediate destruction of tumor cells expressing $\mathrm{GD}_{2}$ via their FcRs, via IL2R-mediated events, or by utilizing both FcRs and IL2Rs simultaneously.

\section{CLINICAL ACTIVITY OF HU14.18-IL2 IC IN NEUROBLASTOMA}

A recently conducted COG NBL phase II study (Shusterman et al., 2010) evaluated the anti-tumor activity and immunological effects of hu14.18-IL2 IC in children with recurrent or refractory disease. A daily dose of $12 \mathrm{mg} / \mathrm{m}^{2}$ was given intravenously for three consecutive days every 28 days. While no responses were noted in the 15 patients with bulky disease detected by computed tomography (CT) scan or magnetic resonance imaging (MRI), 5 of 24 patients with less-bulky disease, evaluable only by bone marrow biopsy or Iodine-131-Meta-Iodobenzylguanidine ( ${ }^{131}$ I-MIBG) scintigraphy, had complete responses. Two other patients with less-bulky disease showed clear evidence of anti-tumor effect, but did not quite meet the stringent response criteria of the study. The dose limiting toxicities of hu14.18-IL2 IC included capillary leak, pain, and allergic reaction (Osenga et al., 2006). The difference between clinical activity in the patient group with bulky disease vs. the group with less-bulky disease was significant $(p=0.03)$ and was consistent with results from preclinical studies in tumor-bearing mice, in which better anti-tumor activity was seen in animals with smaller tumors at the time treatment was initiated (Neal et al., 2004b).

\section{INFLUENCE OF FCR POLYMORPHISMS ON ADCC ACTIVITY}

Studies of people expressing polymorphic variants of FcRs have demonstrated that responses in mAb-based cancer therapy vary depending on FcR polymorphisms. In humans, the $\mathrm{FcR}$ found on NK cells, CD16, displays a single nucleotide polymorphism (SNP) resulting in a different amino acid in the IgG-binding domain at position 158. Either phenylalanine (F) or valine (V) is present, which is associated with a lower or a higher affinity for the Fc portion respectively. Subsequently, studies using the tumor-targeted mAb Rituximab have shown that the V/V genotype has the highest binding affinity to IgG1 isotype and initiates more robust ADCC activity in vitro. Furthermore, a positive correlation has been drawn between a V/V genotype and superior clinical response to Rituximab. In studies of Rituximab in Non-Hodgkin's lymphoma (Cartron et al., 2002; Weng and Levy, 2003), patients with the high affinity V/V genotype showed a higher complete or partial response rate of $96 \%$ compared to $62 \%$ for patients with the intermediate (F/V) or low affinity (F/F) genotypes. Thus, carrying the high affinity CD16 genotype can be used as a positive predictive marker for better response to Rituximab, and likely to other human mAbs of an IgG1 isotype.

Most, but not all, studies on Rituximab, Trastuzumab, and Cetuximab demonstrated greater clinical benefit in patients with high affinity V/V genotypes of CD16 (Cartron et al., 2002; Weng and Levy, 2003;Zhang et al., 2007; Musolino et al., 2008;Taylor et al., 2009). In addition, there is also a benefit of having a genotype for a high affinity CD32, the FcR found on neutrophils and monocytes/macrophages. CD32 also has a SNP in humans consisting of two alleles. These alleles differ in the IgGbinding domain at position 131, where either a lower affinity arginine $(\mathrm{R})$ or a higher affinity histidine $(\mathrm{H})$ is present. For $\mathrm{CD} 32$, the $\mathrm{H} / \mathrm{H}$ genotype has the highest affinity binding to the IgG1 isotype and initiates more robust ADCC in vitro. Similar to $\mathrm{CD} 16$, the high affinity $\mathrm{CD} 32$ subtype $(\mathrm{H} / \mathrm{H})$ has also been associated with a greater clinical response to the above mentioned therapeutic mAbs (Weng and Levy, 2003; Zhang et al., 2007; Musolino et al., 2008; Taylor et al., 2009). Collectively, these studies suggest that the in vivo efficacy of therapeutic mAbs is, at least in part, dependent on the affinity of FcRs for IgG1, consistent with a major role for in vivo ADCC as the mechanism of action.

\section{INHIBITORY KIRS AND NK CELL RESPONSES}

Killer immunoglublin-like receptors are cell surface proteins of NK cells that regulate NK cell activation and function. Inhibitory KIRs are distinguished from activating KIRs by the inclusion of an immunoreceptor tyrosine-based inhibitory motif (ITIM) in the cytoplasmic signaling domain, leading to a potent inhibition of multiple cell processes upon engagement (Purdy and Campbell, 2009; Leung, 2011). While many ligands for activating KIRs are not well established, inhibitory KIRs recognize Class I human leukocyte antigen (HLA-I) molecules (KIR-L), which are expressed by all nucleated cells. The expression of inhibitory KIRs helps prohibit NK effector function against HLA-expressing autologous normal cells (Vilches and Parham, 2002). Downregulation of HLA is a mechanism by which virally infected and transformed cells evade $\mathrm{T}$ cell recognition (Vilches and Parham, 2002). However in the absence of normal HLA-I expression, NK cells are not inhibited through their KIRs, potentially resulting in lysis of autologous cells (Kärre, 2002; Vilches and Parham, 2002). NK cells are often described as natural effector cells against virally infected and transformed autologous cells (Purdy and Campbell, 2009) and much of this responsiveness is dictated by the balance of activating signals with the engagement of inhibitory KIRs (Kärre, 2002; Orr et al., 2010). Thus, the effector function of NK cells is tightly regulated by inhibitory KIR signaling and is of great importance to NK-mediated immunotherapy regimens.

Killer immunoglublin-like receptor in humans bind to specific HLA Class I molecules (KIR-L) coded for by the A, B, and C loci (Velardi, 2008). Four inhibitory KIRs: KIR2DL1, KIR2DL2, KIR2DL3, and KIR3DL1 have received a lot of attention in various cell therapy settings (Purdy and Campbell, 2009). The importance of KIR/KIR-L interaction for the anti-cancer activity of NK cells was demonstrated in the setting of allogeneic hematopoietic stem cell transplant (HSCT). In HLA-haploidentical transplantation patients with acute myeloid leukemia (AML), Ruggeri et al. evaluated the response of leukemia patients where the HLA Class I expression of KIR ligands (KIR-L) on donor and recipient indicated that donor NK cells might not all be inhibited by recipient HLA (designated as "KIR-Ligand incompatibility" in the "GVH 
direction"). In this analysis, patients with AML were found to have a survival advantage if they had a KIR-Ligand incompatibility. Five years post transplant $100 \%$ of AML patients with a missing KIR-L were disease free compared to $25 \%$ of patients whose tissues expressed KIR-Ls for all donor NK cells (Ruggeri et al., 2002). An update from Ruggeri et al. (2007) further demonstrated the benefit of KIR-L incompatibility in AML patients after transplant. Of the 112 HLA-haploidentical transplants in high-risk AML patients, those who were KIR-L incompatible for their transplant had significantly better event-free survival compared to patients without KIR-L incompatibility $(67 \% ; n=51$ vs. $18 \% ; n=61$ respectively; $p=0.02$ ). Thus, improved leukemia control can be conferred by alloreactive donor NK cells, in AML patients whose cells are KIR$\mathrm{L}$ incompatible in the GVH direction. In contrast, in these same reports, there was no benefit noted for a missing KIR-L in patients with acute lymphoid leukemia (ALL).

More recently, Leung et al. (2007) proposed a separate way of analyzing these allogeneic KIR relationships. By first characterizing the genotype for the inhibitory KIRs of the donor (KIR2DL1, KIR2DL2, KIR2DL3, and KIR3DL1), and then genotyping the HLA Class I KIR ligands of the recipient, one could determine whether donor NK cells contained at least one KIR for which the recipient lacked a corresponding HLA-ligand ("missing KIR-L," or "KIR/KIR-Ligand mismatch"). In those transplant recipients with the "missing KIR-L," there was at least one inhibitory KIR on some of the transplanted NK cells that could not be ligated by the HLA-I molecules on the patient's cells. In this analysis, pediatric patients with AML or ALL were found to have a survival advantage if they and their allogeneic donor were KIR/KIR-L mismatched (Leung et al., 2004).

The initial clinical analyses of KIR/KIR-L mismatch were restricted to allogeneic HSCT (Ruggeri et al., 2002; Hsu et al., 2005; Vivier et al., 2011) and allogeneic adoptive NK infusions (Miller et al., 2005; Bachanova et al., 2010;Geller et al., 2011). KIR genes and HLA-I genes are inherited independently because they are encoded on different chromosomes (19q13.4 and 6p21.3 respectively; Purdy and Campbell, 2009). Moreover, there is a great degree of polymorphism of these genes among individuals (Vilches and Parham, 2002; Purdy and Campbell, 2009). The independent segregation and stochastic expression of KIR and HLA-I genes can cause some individuals to have NK cells that express KIRs for which that same individual has no corresponding HLA (KIR-L). This situation has been designated by Leung and colleagues as KIR/KIR-L mismatch. Various reports of clinical trials have suggested that an autologous KIR/KIR-L mismatch occurs in approximately $60 \%$ of patients (Moretta and Moretta, 2004; Leung et al., 2007; Venstrom et al., 2009; Delgado et al., 2010). Leung et al. (2007) demonstrated a clinical benefit of autologous KIR/KIR-L mismatch in pediatric patients that received ASCT for lymphoma or solid tumors . Autologous KIR/KIR-L mismatch has been implicated as a favorable prognostic factor in high-risk NBL patients following autologous HSCT. In a retrospective study of 169 patients, Venstrom et al. (2009) found a significantly lower risk of death and disease progression in patients lacking one or more autologous KIR-L ( 46 and 34\%, with $p=0.007$ and $p=0.047$ respectively) compared to patients who were fully autologous KIR/KIR-L matched.

\section{GENOTYPING FOR FAVORABLE KIR AND FCR ALLELES IN PATIENTS RECEIVING HU14.18-IL2}

Our lab has shown that hu14.18-IL2 IC therapy in mice elicits NKmediated anti-tumor responses that can be independent of $\mathrm{T}$ cells (Neal et al., 2004b). We have also shown that murine NBL cells can escape hu14.18-IL2 IC therapy by increasing MHC-I expression, a mechanism capable of down regulating NK cell function via the mouse equivalent of inhibitory KIRs - the inhibitory Ly49 receptors (Neal et al., 2004a). Furthermore, the advantage of KIR/KIR-L mismatch in allogeneic and autologous transplant setting has been demonstrated (Ruggeri et al., 2002; Leung et al., 2007; Venstrom et al., 2009). These observations led us to hypothesize that individuals with an inherited autologous KIR/KIR-L mismatch would respond better to hu14.18-IL2 IC-mediated NK-based cancer immunotherapy than those without. We further hypothesized, based on the known importance of FcR polymorphisms for higher affinity alleles in $\mathrm{mAb}$ therapy, that we would see a correlation between responses to hu14.18-IL2 IC immunotherapy and genotypes for high affinity polymorphisms for both CD32 and CD16 FcRs.

To test these hypotheses our lab analyzed the relationship between FcR genotype and KIR/KIR-L match or mismatch status with regard to the clinical response of patients following hu14.18IL2 IC therapy using patient samples from the phase II COG NBL study (Delgado et al., 2010). We performed FcR and KIR/KIR-L genotyping on DNA isolated from clinical samples from patients enrolled in this study $(n=38)$. For these analyses, an individual's $\mathrm{KIR} / \mathrm{KIR}-\mathrm{L}$ status is determined by genotyping for the presence or absence of inhibitory KIR genes (2DL1, 2DL2, 2DL3, and 3DL1) as well as their ligands, allelic groups of HLA genes: HLA-C1, HLA-C2, and HLA-Bw4. The detection of inhibitory KIR genes is currently done in our laboratory by a SYBR Green Real Time PCR reaction with primers designed to amplify a specific gene locus followed by analysis of the melting curve (Vilches and Parham, 2002; Alves et al., 2009; Hong et al., 2011). While the complexity of the polymorphism of HLA genes is tremendous, the recognition by KIRs distinguishes families of HLA alleles in a relatively simple, bi-allelic fashion. In order to specifically address the broad HLA specificities (HLA-C1, HLA-C2, and HLA-Bw4) as recognized by KIRs, typing methods include PCR amplification with sequencespecific primers (PCR-SSP), PCR amplification using a sequencespecific oligonucleotide probe (PCR-SSOP), and sequence-based typing (SBT). Additionally, SYBR Green Real Time PCR methods, similar to that described above for KIR genotyping, can be used to determine HLA-C1, HLA-C2, and HLA-Bw4 gene status (Hong et al., 2011).

When we evaluated the KIR/KIR-L genotype of patients, we found a statistically significant difference $(p=0.03)$ between the outcome of patients that had an autologous KIR/KIR-L mismatch and those that were KIR/KIR-L matched. Upon closer evaluation, our data showed that of the seven patients that showed improvement, all seven inherited an autologous KIR/KIR-L mismatch. Although our study was small, we believe this to be a novel demonstration of autologous KIR/KIR-L status as a predictor of response to immunotherapy beyond that shown previously in the setting of adoptive allogeneic BMT or NK cell transfer or autologous HSCT. 
We also evaluated FcR genotypes in the same cohort of patients in our small phase II study of hu14.18-IL2 IC (Delgado et al., 2010; Shusterman et al., 2010). In order to evaluate FcR genotype, analyses of SNP in the Fc receptor genes FcgR2A 131-H/R (rs1801274) and FcgR3A 158-F/V (rs396991) are performed using pyrosequencing and/or Taqman probes available from Applied Biosystems (Delgado et al., 2010). We found a trend toward better response in individuals that had high affinity CD32 alleles $(p=0.06)$. These analyses suggest that high affinity CD32 FcRs on neutrophils and monocytes may enhance their antibodydependent anti-tumor response. In contrast there was no trend noted with high affinity alleles for CD16 $(p=1.0)$. This may have been due to poor statistical power, as only 2 of the 36 patients typed for CD16 showed the high affinity CD16 V/V genotype. Moreover, when we compared homozygotes for high affinity receptors together with heterozygotes $[\mathrm{V} / \mathrm{V}+\mathrm{V} / \mathrm{F}(n=19)]$ to those patients that were homozygous for low affinity genotypes [F/F $(n=17)]$ we were unable to draw a significant correlation with response ( $p=0.41$; unpublished analysis). Given these data and due to the small sample size of our phase II study of hu14.18-IL2 IC, the study could not determine whether there was any interaction between KIR/KIR-L match or mismatch status and FcR genotype for CD16 or CD32.

Our preclinical data suggesting that IL2 and IL2Rs play a role in the immune synapses that form between NK cells and tumor cells coated with hu14.18-IL2 (Buhtoiarov et al., 2011; Gubbels et al., 2011) may explain the apparent lack of importance of high affinity CD16 in response to hu14.18-IL2. In vitro, hu14.18-IL2 IC can mediate conjugation of NKL cells to tumor cells (Figure 1). Upon IC treatment, the Fab portion of the IC binds to the antigenic target $\left(\mathrm{GD}_{2}\right.$ in the case of 14.18-IL2 IC) on tumor cells and the IL2 component of the IC interacts with IL2Rs on effector cells bridging the two cells. This bridging results in the formation of an AIS defined by the polarization of effector molecules including adhesion molecules and IL2Rs on the NKL cells to the immune synapse and the subsequent hu14.18-IL2 IC-facilitated NK cellmediated tumor cell killing (Gubbels et al., 2011). It is possible that the beneficial effect of high affinity CD16 alleles may be less important with IC than with a conventional mAb. Namely the conventional mAb binds to tumor and then interacts with effectors only through cell-bound IgG interacting with FcR on effector cells; in contrast the IC causes changes to the immune synapse via IL2/IL2R interactions in addition to Fc/FcR interactions, and also activates the NK cell by localized IL2. As such, as long as IL2R are present on the effector cells, even low affinity FcR may be sufficient for potent immune synapse formation.

\section{HYPOTHESES}

Based on the data evaluating the roles of KIR/KIR-L and FcR genotypes from our small phase II study of hu14.18-IL2 IC in NBL patients (Delgado et al., 2010; Shusterman et al., 2010), we hypothesize that the anti-tumor effect of $\mathrm{mAb}$ treatment, designed to mediate ADCC in vivo, will be more productive in individuals with a favorable KIR/KIR-L mismatch genotype. We also anticipate that data will continue to show $\mathrm{mAb}$ treatment is more beneficial for patients with higher affinity genotypes for CD32 and CD16. Furthermore, we hypothesize that the best responses might be seen in individuals that have both favorable KIR/KIR$\mathrm{L}$ and high affinity FcR genotypes. Figure $\mathbf{2}$ is a schematic that depicts our hypotheses. We believe that: (a) having both high affinity FcRs and being autologous KIR/KIR-L mismatched would result in the best response/greatest tumor cell lysis; (b) having low affinity FcRs and being KIR/KIR-L matched would result in the poorest response/least amount of lysis; and (c) having either high affinity FcRs and being KIR/KIR-L matched or having low affinity FcRs and being KIR/KIR-L mismatched would result in an intermediate response/level of lysis. It is important to note these hypotheses are not limited to anti-GD2 mAb therapy in the context of NBL. The similar effects of $F c \gamma R$ polymorphisms on the anti-tumor effects of other cancer-reactive mAbs in clinical use (i.e., Rituxan, Trastuzumab, Cetuximab) suggest that patients with a favorable genotype may be selected for any mAb-based tumor therapy in which ADCC is a possible mechanism. We hypothesize that KIR/KIR-L relationships may also prove to be predictive of response for the anti-tumor effects of these other mAbs as well.

There are currently few predictive biomarkers to identify cancer patients most likely to respond to mAb therapy. In breast cancer, Trastuzumab is indicated for adjuvant and palliative treatment and its use is restricted to the approximately $25 \%$ of patients whose tumors express high levels of the HER2 receptor (Adams and Weiner, 2005). Despite limiting Trastuzumab's use for HER2 positive tumors, the response rate for Trastuzumab monotherapy ranges from just 11 to 26\% (Dougan and Dranoff, 2009). Further improvements in selecting patients likely to respond to Trastuzumab are warranted. In the setting of chemorefractory colorectal cancer (CRC), K-ras mutations serve as a biomarker predicting response to Cetuximab. CRC patients with tumors that have K-ras mutations realized no significant survival benefit from Cetuximab whereas patients with wild-type K-ras tumors achieved longer progression free- and overall survival than best supportive care alone (Karapetis et al., 2008). However, for CRC patients with wild-type K-ras tumors, the response rate is still less than $14 \%$ (Karapetis et al., 2008) highlighting the need for additional factors predicting $\mathrm{mAb}$ anti-tumor efficacy in CRC. In non-small-cell lung cancer, K-ras mutational status does not predict benefit from Cetuximab therapy (Khambata-Ford et al., 2010; O'Byrne et al., 2011) and the potential utility of using FcR and KIR/KIR-L genotype status to predict Cetuximab efficacy could impact therapeutic decision-making for thousands of NSCLC patients in the US each year.

In order to address whether or not $\mathrm{FcR}$ and KIR/KIR-L genotyping can be used as predictive markers for favorable therapeutic outcome, genotyping needs to be done for large clinical-ADCCbased $\mathrm{mAb}$ immunotherapy trials that have adequate numbers of patients enrolled and sufficient numbers of patients that benefited from the therapy. Such large analyses should have the statistical power necessary to test whether favorable FcR and KIR/KIR-L genotypes interact to augment the anti-tumor effect mediated by $\mathrm{mAbs}$ via ADCC. Furthermore, additional analyses of larger clinical trials using ICs in which the mAb is directly linked to IL2 are needed in order to determine whether the added interactions mediated by IL2Rs on NK cells can provide substantial benefit, thereby circumventing the disadvantage for low affinity CD16 on the NK cells. 


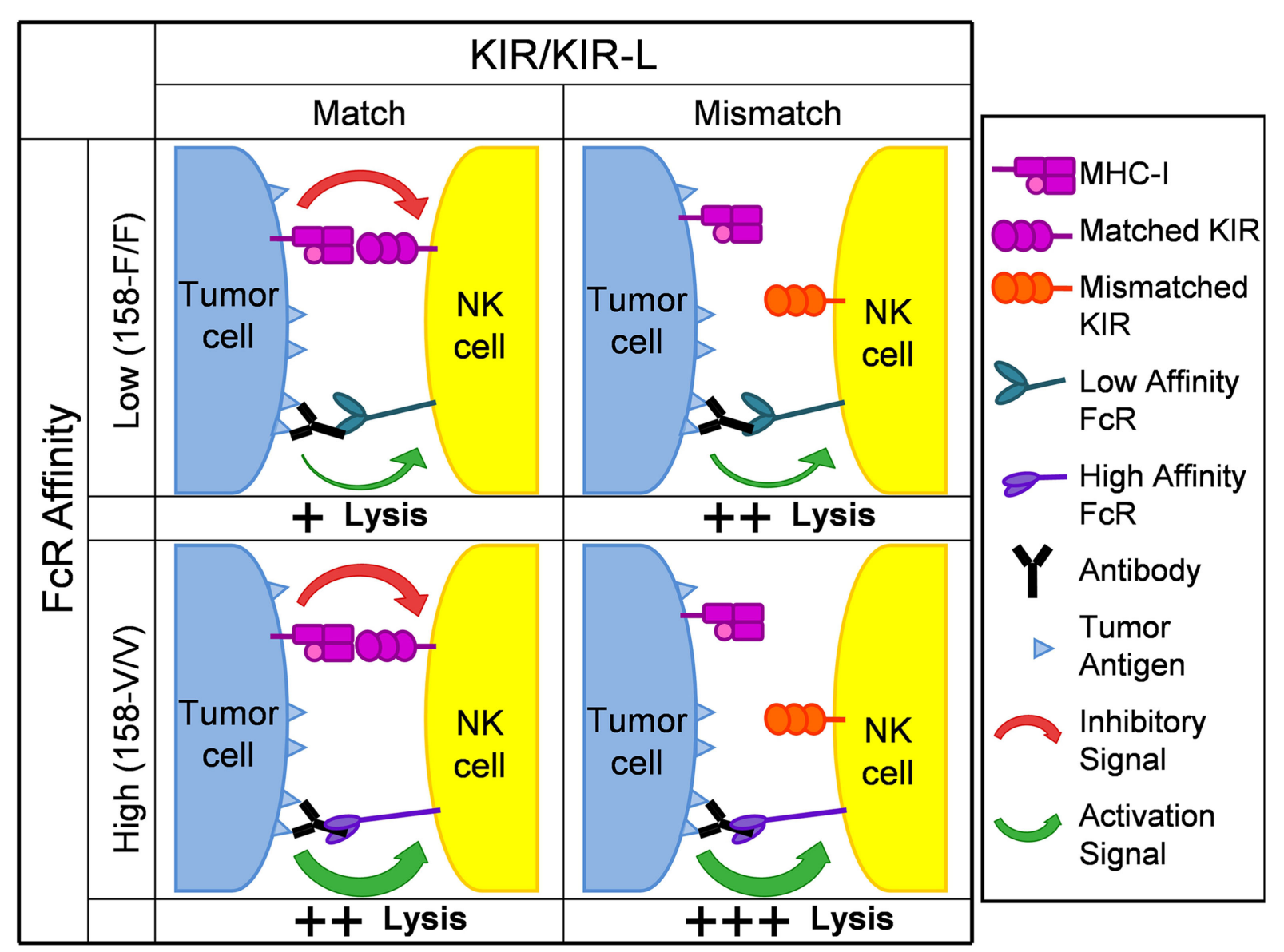

FIGURE 2 | Schematic depicting hypothesized impact of KIR/KIR-L and FcR genotype on anti-tumor activity following mAb treatment. KIR molecules are shown as either being matched or mismatched for the appropriate Class I Major Histocompatibility Complex (MHC) antigen on the tumor cell (HLA Class I in humans). The FcR genotypes shown correspond to those for CD16 on NK cells. For any potential synapse between an NK cell and a tumor cell, multiple interactions between many Fc components on mAb-coated tumor cells and many CD16 molecules on the NK cell (similar to the individual interaction shown) would need to be involved. Similarly, multiple interactions between multiple HLA Class I molecules on the tumor cell and multiple KIR receoptors on the NK cell (similar to the individual interaction shown) would also be involved. Analogous relationships are predicted for the interaction of $\mathrm{FC}$ and the distinct $\mathrm{CD} 32$ polymorphisms expressed on neutrophils, monocytes and macrophages. The overall response will reflect the balancing of the signal activating tumor lysis (depicted as a green arrow) with the inhibitory effect of KIR ligation (depicted as a red arrow). The poorest response/least tumor cell lysis (designated + ) would result from having low affinity FcRs and being KIR/KIR-L matched (upper left quadrant). The best response/greatest tumor cell lysis (designated +++ ) would result from having high affinity FcRs and being autologous KIR/KIR-L mismatched (lower right quadrant). Having either low affinity FcRs and being KIR/KIR-L mismatched (upper right quadrant) or having high affinity FcRs and being KIR/KIR-L matched (lower left quadrant) would result in an intermediate response/level of tumor cell lysis (designated ++ ).

\section{CONCLUSION}

Recent advances in immunotherapeutic treatment for high-risk NBL have shown clinical benefit. Additional research is needed to develop therapeutic options to effectively eradicate the disease in those patients who are not currently being cured. Tumor cell recognition and subsequent signaling utilized by NK cells are multi-modal and complex. However, as our understanding of NK cell biology advances, so does the clinical benefit realized in the applied field of cancer immunotherapy. One such example is our relatively nascent understanding of the clinical implications of NK cell receptor biology, in particular the KIR/KIR-L relationships. As the evidence for the importance of KIR/KIR-L and FcR genotypes continues to mount, there exists an opportunity to apply our understanding of these relationships in the context of hu14.18-IL2 IC-mediated NK-based cancer immunotherapy for improved NBL treatment. These principles, and the potential interactions between KIR/KIR-L relationships and FcR genotypes may also apply (and are testable) in the clinical development of other tumor-reactive mAbs and ICs that mediate clinically meaningful ADCC against other more common cancers.

\section{ACKNOWLEDGMENTS}

We thank Jane Kolstad and Mary Maas for administrative assistance. This work was supported by National Institutes of Health Grants CA032685, CA87025, CA14520, GM067386, and grants from the Midwest Athletes for Childhood Cancer Fund, the Crawdaddy Foundation, The Evan Dunbar Foundation, the UW-Cure 
Kids Cancer Coalition, Abbie's Fund, The Hyundai Hope on Wheels Program and the Super Jake Foundation (to Paul M. Sondel). Tony A. Koehn and L.S. were supported by the National Institutes of Health Pre-doctoral Training Grant T32 GM007215

\section{REFERENCES}

Adams, G. P., and Weiner, L. M. (2005). Monoclonal antibody therapy of cancer. Nat. Biotechnol. 23, $1147-1157$.

Alvarez-Rueda, N., Desselle, A., Cochonneau, D., Chaumette, T., Clemenceau, B., Leprieur, S., Bougras, G., Supiot, S., Mussini, J. M., Barbet, J., Saba, J., Paris, F., Aubry, J., and Birkle, S. (2011). A monoclonal antibody to O-acetyl-GD2 ganglioside and not to GD2 shows potent anti-tumor activity without peripheral nervous system crossreactivity. PLoS ONE 6, e25220. doi:10.1371/journal.pone. 0025220

Alves, L. G., Rajalingam, R., and Canavez, F. (2009). A novel real-time PCR method for KIR genotyping. Tissue Antigens 73, 188-191.

Bachanova, V., Burns, L. J., Mckenna, D. H., Curtsinger, J., PanoskaltsisMortari, A., Lindgren, B. R., Cooley, S., Weisdorf, D., and Miller, J. S. (2010). Allogeneic natural killer cells for refractory lymphoma. Cancer Immunol. Immunother. 59, 1739-1744.

Basham, T. Y., Race, E. R., Campbell, M. J., Reid, T. R., Levy, R., and Merigan, T. C. (1988). Synergistic antitumor activity with IFN and monoclonal anti-idiotype for murine B cell lymphoma. Mechanism of action. J. Immunol. 141, 2855-2860.

Becknell, B., and Caligiuri, M. A. (2008). Natural killer cells in innate immunity and cancer. J. Immunother. 31, 685-692.

Biron, C. A., Nguyen, K. B., Pien, G. C., Cousens, L. P., and Salazar-Mather, T. P. (1999). Natural killer cells in antiviral defense: function and regulation by innate cytokines. Annu. Rev. Immunol. 17, 189-220.

Borgerding, A., Hasenkamp, J., Engelke, M., Burkhart, N., Trümper, L., Wienands, J., and Glass, B. (2010). B-lymphoma cells escape rituximab-triggered elimination by NK cells through increased HLA class I expression. Exp. Hematol. 38, 213-221.

Buhtoiarov, I. N., Neal, Z. C., Gan, J., Buhtoiarova, T. N., Patankar, M. S., Gubbels, J. A., Hank, J. A., Yamane, B., Rakhmilevich, A. L., Reisfeld, R. A., Gillies, S. D., and Sondel, P. M. (2011). Differential internalization of hul4.18-IL2 immunocytokine by NK and tumor cell: impact on conjugation, cytotoxicity, and targeting. J. Leukoc. Biol. 89, 625-638.

Cartron, G., Dacheux, L., Salles, G., Solal-Celigny, P., Bardos, P., Colombat, P., and Watier, H. (2002). Therapeutic activity of humanized antiCD20 monoclonal antibody and polymorphism in IgG Fc receptor FcgammaRIIIa gene. Blood 99, 754-758.

Clynes, R. A., Towers, T. L., Presta, L. G., and Ravetch, J. V. (2000). Inhibitory $\mathrm{Fc}$ receptors modulate in vivo cytotoxicity against tumor targets. Nat. Med. 6, 443-446.

Delgado, D. C., Hank, J. A., Kolesar, J., Lorentzen, D., Gan, J., Seo, S., D., Reisfeld, R. A., Yang, R., Gadbaw, B., Desantes, K. B., London, W. B., Seeger, R. C., Maris, J. M., and Sondel, P. M. (2010). Genotypes of NK cell KIR receptors, their ligands, and Fc $\gamma$ receptors in the response of neuroblastoma patients to Hu14.18IL2 immunotherapy. Cancer Res. 70, 9554-9561.

Dougan, M., and Dranoff, G. (2009). Immune therapy for cancer. Annu. Rev. Immunol. 27, 83-117.

Farag, S. S., and Caligiuri, M. A. (2006). Human natural killer cell development and biology. Blood Rev. 20, 123-137.

Farag, S. S., Vandeusen, J. B., Fehniger, T. A., and Caligiuri, M. A. (2003). Biology and clinical impact of human natural killer cells. Int. J. Hematol. 78, 7-17.

Geller, M. A., Cooley, S., Judson, P. L., Ghebre, R., Carson, L. F., Argenta, P. A., Jonson, A. L., PanoskaltsisMortari, A., Curtsinger, J., Mckenna, D., Dusenbery, K., Bliss, R., Downs, L. S., and Miller, J. S. (2011). A phase II study of allogeneic natural killer cell therapy to treat patients with recurrent ovarian and breast cancer. Cytotherapy 13, 98-107.

Gillies, S. D., Lan, Y., Williams, S., Carr, F., Forman, S., Raubitschek, A., and Lo, K. M. (2005). An anti-CD20-IL-2 immunocytokine is highly efficacious in a SCID mouse model of established human B lymphoma. Blood 105, 3972-3978.

Gillies, S. D., Reilly, E. B., Lo, K. M., targeted interleukin 2 stimulates Tcell killing of autologous tumor cells. Kim, K., Shusterman, S., Gillies, S. and Reisfeld, R. A. (1992). Antibody-

in Molecular Biosciences from the National Institute of General Medical Sciences and Advanced Opportunity Fellowship through SciMed Graduate Research Scholars at the University of Wisconsin-Madison.

Proc. Natl. Acad. Sci. U.S.A. 89, 1428-1432.

Gubbels, J. A., Gadbaw, B., Buhtoiarov, I. N., Horibata, S., Kapur, A. K., Patel, D., Hank, J. A., Gillies, S. D., Sondel, P. M., Patankar, M. S., and Connor, J. (2011). Ab-IL2 fusion proteins mediate NK cell immune synapse formation by polarizing CD25 to the target cell-effector cell interface. Cancer Immunol. Immunother. 60, 1789-1800.

Hank, J. A., Albertini, M. R., Schiller, J., and Sondel, P. M. (1993). Activation of multiple effector mechanisms to enhance tumor immunotherapy. J. Immunother. Emphasis Tumor Immunol. 14 329-335.

Hank, J. A., Kohler, P. C., WeilHillman, G., Rosenthal, N., Moore, K. H., Storer, B., Minkoff, D., Bradshaw, J., Bechhofer, R., and Sondel, P. M. (1988). In vivo induction of the lymphokine-activated killer phenomenon: interleukin 2dependent human non-major histocompatibility complex-restricted cytotoxicity generated in vivo during administration of human recombinant interleukin 2. Cancer Res. 48, 1965-1971.

Hank, J. A., Robinson, R. R., Surfus, J., Mueller, B. M., Reisfeld, R. A., Cheung, N. K., and Sondel, P. M. (1990). Augmentation of antibody dependent cell mediated cytotoxicity following in vivo therapy with recombinant interleukin 2. Cancer Res. 50, 5234-5239.

Hong, H. A., Loubser, A. S., De Assis Rosa, D., Naranbhai, V., Carr W., Paximadis, M., Lewis, D. A., Tiemessen, C. T., and Gray, C. M. (2011). Killer-cell immunoglobulinlike receptor genotyping and HLA killer-cell immunoglobulin-like receptor-ligand identification by real-time polymerase chain reaction. Tissue Antigens 78, 185-194.

Hsu, K. C., Keever-Taylor, C. A., Wilton, A., Pinto, C., Heller, G., Arkun, K., O'Reilly, R. J., Horowitz, M. M. and Dupont, B. (2005). Improved outcome in HLA-identical sibling hematopoietic stem-cell transplantation for acute myelogenous leukemia predicted by KIR and HLA genotypes. Blood 105, 4878-4884.

Huenecke, S., Zimmermann, S. Y., Kloess, S., Esser, R., Brinkmann, A., Tramsen, L., Koenig, M., Erben,
S., Seidl, C., Tonn, T., Eggert, A., Schramm, A., Bader, P., Klingebiel, T., Lehrnbecher, T., Passweg, J. R., Soerensen, J., Schwabe, D., and Koehl, U. (2010). IL-2-driven regulation of NK cell receptors with regard to the distribution of CD16+ and CD16- subpopulations and in vivo influence after haploidentical NK cell infusion. J. Immunother. 33, 200-210.

Jefferis, R. (2007). Antibody therapeutics: isotype and glycoform selection. Expert Opin. Biol. Ther. 7, 1401-1413.

Johnson, E., Dean, S. M., and Sondel, P. M. (2007). Antibody-based immunotherapy in high-risk neuroblastoma. Expert Rev. Mol. Med. 9, $1-21$.

Karapetis, C. S., Khambata-Ford, S., Jonker, D. J., O'Callaghan, C. J., Tu, D., Tebbutt, N. C., Simes, R. J., Chalchal, H., Shapiro, J. D., Robitaille, S., Price, T. J., Shepherd, L., Au, H. J., Langer, C., Moore, M. J., and Zalcberg, J. R. (2008). K-ras mutations and benefit from cetuximab in advanced colorectal cancer. N. Engl. J. Med. 359, $1757-1765$

Kärre, K. (2002). NK cells, MHC class I molecules and the missing self. Scand. J. Immunol. 55, 221-228.

Khambata-Ford, S., Harbison, C. T., Hart, L. L., Awad, M., Xu, L. A., Horak, C. E., Dakhil, S., Hermann, R. C., Lynch, T. J., and Weber, M. R. (2010). Analysis of potential predictive markers of cetuximab benefit in BMS099, a phase III study of cetuximab and first-line taxane/carboplatin in advanced nonsmall-cell lung cancer. J. Clin. Oncol. 28, 918-927.

Leung, W. (2011). Use of NK cell activity in cure by transplant. Br. J. Haematol. 155, 14-29.

Leung, W., Handgretinger, R., Iyengar, R., Turner, V., Holladay, M. S., and Hale, G. A. (2007). Inhibitory KIR-HLA receptor-ligand mismatch in autologous haematopoietic stem cell transplantation for solid tumour and lymphoma. Br. J. Cancer 97, 539-542.

Leung, W., Iyengar, R., Turner, V., Lang, P., Bader, P., Conn, P., Niethammer, D., and Handgretinger, R. (2004). Determinants of antileukemia effects of allogeneic NK cells. J. Immunol. 172, 644-650. 
Lode, H. N., Xiang, R., Becker, J. C., Gillies, S. D., and Reisfeld, R. A. (1998a). Immunocytokines: a promising approach to cancer immunotherapy. Pharmacol. Ther. 80, 277-292.

Lode, H. N., Xiang, R., Dreier, T., Varki, N. M., Gillies, S. D., and Reisfeld, R. A. (1998b). Natural killer cellmediated eradication of neuroblastoma metastases to bone marrow by targeted interleukin-2 therapy. Blood 91, 1706-1715.

Miller, J. S., Soignier, Y., PanoskaltsisMortari, A., Mcnearney, S. A., Yun, G. H., Fautsch, S. K., Mckenna, D., Le, C., Defor, T. E., Burns, L. J., Orchard, P. J., Blazar, B. R., Wagner, J. E., Slungaard, A., Weisdorf, D. J., Okazaki, I. J., and Mcglave, P. B. (2005). Successful adoptive transfer and in vivo expansion of human haploidentical NK cells in patients with cancer. Blood 105, 3051-3057.

Modak, S., and Cheung, N. K. (2007). Disialoganglioside directed immunotherapy of neuroblastoma. Cancer Invest. 25, 67-77.

Moretta, L., Bottino, C., Pende, D., Vitale, M., Mingari, M. C., and Moretta, A. (2005). Human natural killer cells: Molecular mechanisms controlling NK cell activation and tumor cell lysis. Immunol. Lett. 100, 7-13.

Moretta, L., and Moretta, A. (2004). Killer immunoglobulin-like receptors. Curr. Opin. Immunol. 16, 626-633.

Musolino, A., Naldi, N., Bortesi, B., Pezzuolo, D., Capelletti, M., Missale, G., Laccabue, D., Zerbini, A., Camisa, R., Bisagni, G., Neri, T. M., and Ardizzoni, A. (2008). Immunoglobulin $\mathrm{G}$ fragment $\mathrm{C}$ receptor polymorphisms and clinical efficacy of trastuzumab-based therapy in patients with HER-2/neu-positive metastatic breast cancer. J. Clin. Oncol. 26, 1789-1796.

Navid, F., Armstrong, M., and Barfield, R. C. (2009). Immune therapies for neuroblastoma. Cancer Biol. Ther. 8, 874-882.

Neal, Z. C., Imboden, M., Rakhmilevich, A. L., Kim, K. M., Hank, J. A., Surfus, J., Dixon, J. R., Lode, H. N., Reisfeld, R. A., Gillies, S. D., and Sondel, P. M. (2004a). NXS2 murine neuroblastomas express increased levels of MHC class I antigens upon recurrence following NK-dependent immunotherapy. Cancer Immunol. Immunother. 53, 41-52.

Neal, Z. C., Yang, J. C., Rakhmilevich, A. L., Buhtoiarov, I. N., Lum, H. E., Imboden, M., Hank, J. A., Lode, H. N., Reisfeld, R. A., Gillies, S. D., and Sondel, P. M. (2004b). Enhanced activity of hu14.18-IL2 immunocytokine against murine NXS2 neuroblastoma when combined with interleukin 2 therapy. Clin. Cancer Res. 10, 4839-4847.

Nimmerjahn, F., and Ravetch, J. V. (2008). Fcgamma receptors as regulators of immune responses. Nat. Rev. Immunol. 8, 34-47.

O’Byrne, K. J., Gatzemeier, U., Bondarenko, I., Barrios, C., Eschbach, C., Martens, U. M., Hotko, Y., Kortsik, C., Paz-Ares, L., Pereira, J. R., Von Pawel, J., Ramlau, R., Roh, J. K., Yu, C. T., Stroh, C., Celik, I., Schueler, A., and Pirker, R. (2011). Molecular biomarkers in non-small-cell lung cancer: a retrospective analysis of data from the phase 3 FLEX study. Lancet Oncol. 12, 795-805.

Oliva, A., Kinter, A. L., Vaccarezza, M. Rubbert, A., Catanzaro, A., Moir, S., Monaco, J., Ehler, L., Mizell, S., Jackson, R., Li, Y., Romano, J. W. and Fauci, A. S. (1998). Natural killer cells from human immunodeficiency virus (HIV)-infected individuals are an important source of CC-chemokines and suppress HIV-1 entry and replication in vitro. J. Clin. Invest. 102, 223-231.

Orange, J. S. (2008). Formation and function of the lytic NK-cell immunological synapse. Nat. Rev. Immunol. 8, 713-725.

Orange, J. S., Harris, K. E., Andzelm, M. M., Valter, M. M., Geha, R. S., and Strominger, J. L. (2003). The mature activating natural killer cell immunologic synapse is formed in distinct stages. Proc. Natl. Acad. Sci. U.S.A. 100, 14151-14156.

Orr, M. T., Murphy, W. J., and Lanier, L. L. (2010). 'Unlicensed' natural killer cells dominate the response to cytomegalovirus infection. Nat. Immunol. 11, 321-327.

Osenga, K. L., Hank, J. A., Albertini, M. R., Gan, J., Sternberg, A. G., Eickhoff, J., Seeger, R. C., Matthay, K. K., Reynolds, C. P., Twist, C., Krailo, M., Adamson, P. C., Reisfeld, R. A., Gillies, S. D., Sondel, P. M., and Group, C. S. O. (2006). A phase I clinical trial of the hu14.18-IL2 (EMD 273063) as a treatment for children with refractory or recurrent neuroblastoma and melanoma: a study of the Children's Oncology Group. Clin. Cancer Res. 12, 1750-1759.

Primus, F. J., Finch, M. D., Wetzel, S. A., Masci, A. M., Schlom, J., and Kashmiri, S. V. (1994). Monoclonal antibody gene transfer. Implications for tumor-specific cellmediated cytotoxicity. Ann. N. Y.
Acad. Sci. 716, 154-165; discussion 165-156.

Purdy, A. K., and Campbell, K. S. (2009). Natural killer cells and cancer: regulation by the killer cell Ig-like receptors (KIR). Cancer Biol. Ther. 8, 2211-2220.

Ravetch, J. V., and Bolland, S. (2001). IgG Fc receptors. Annu. Rev. Immunol. 19, 275-290.

Ruggeri, L., Capanni, M., Urbani, E., Perruccio, K., Shlomchik, W. D. Tosti, A., Posati, S., Rogaia, D., Frassoni, F., Aversa, F., Martelli, M. F. and Velardi, A. (2002). Effectiveness of donor natural killer cell alloreactivity in mismatched hematopoietic transplants. Science 295, 2097-2100.

Ruggeri, L., Mancusi, A., Capanni, M., Urbani, E., Carotti, A., Aloisi, T., Stern, M., Pende, D., Perruccio, K. Burchielli, E., Topini, F., Bianchi, E., Aversa, F., Martelli, M. F., and Velardi, A. (2007). Donor natural killer cell allorecognition of missing self in haploidentical hematopoietic transplantation for acute myeloid leukemia: challenging its predictive value. Blood 110, 433-440.

Schrama, D., Reisfeld, R. A., and Becker, J. C. (2006). Antibody targeted drugs as cancer therapeutics. Nat. Rev. Drug Discov. 5, 147-159.

Shusterman, S., London, W. B., Gillies, S. D., Hank, J. A., Voss, S. D., Seeger, R. C., Reynolds, C. P., Kimball, J., Albertini, M. R., Wagner, B., Gan, J., Eickhoff, J., Desantes, K. B., Cohn, S. L., Hecht, T., Gadbaw, B., Reisfeld, R. A., Maris, J. M. and Sondel, P. M. (2010). Antitumor activity of hu14.18-IL2 in patients with relapsed/refractory neuroblastoma: a Children's Oncology Group (COG) phase II study. J. Clin. Oncol. 28, 4969-4975.

Simon, T., Hero, B., Faldum, A., Handgretinger, R., Schrappe, M., Klingebiel, T., and Berthold, F. (2011). Long term outcome of high-risk neuroblastoma patients after immunotherapy with antibody ch14.18 or oral metronomic chemotherapy. BMC Cancer 11, 21. doi:10.1186/1471-2407-11-21

Simon, T., Hero, B., Faldum, A., Handgretinger, R., Schrappe, M., Niethammer, D., and Berthold, F. (2004). Consolidation treatment with chimeric anti-GD2-antibody ch14.18 in children older than 1 year with metastatic neuroblastoma. J. Clin. Oncol. 22, 3549-3557.

Sosman, J. A., Hank, J. A., and Sondel, P. M. (1990). In vivo activation of lymphokine-activated killer activity with interleukin-2: prospects for combination therapies. Semin.
Oncol. 17, 22-30; discussion 38-41.

Taylor, R. J., Chan, S. L., Wood, A., Voskens, C. J., Wolf, J. S., Lin, W., Chapoval, A., Schulze, D. H., Tian, G., and Strome, S. E. (2009). FcgammaRIIIa polymorphisms and cetuximab induced cytotoxicity in squamous cell carcinoma of the head and neck. Cancer Immunol. Immunother. 58, 997-1006.

Velardi, A. (2008). Role of KIRs and KIR ligands in hematopoietic transplantation. Curr. Opin. Immunol. 20, 581-587.

Venstrom, J. M., Zheng, J., Noor, N., Danis, K. E., Yeh, A. W., Cheung, I. Y., Dupont, B., O’Reilly, R. J., Cheung, N. K., and Hsu, K. C. (2009). KIR and HLA genotypes are associated with disease progression and survival following autologous hematopoietic stem cell transplantation for high-risk neuroblastoma. Clin. Cancer Res. 15, 7330-7334.

Vilches, C., and Parham, P. (2002). KIR: diverse, rapidly evolving receptors of innate and adaptive immunity. Annu. Rev. Immunol. 20, 217-251.

Vivier, E., Raulet, D. H., Moretta, A., Caligiuri, M. A., Zitvogel, L., Lanier, L. L., Yokoyama, W. M., and Ugolini, S. (2011). Innate or adaptive immunity? The example of natural killer cells. Science 331, 44-49.

Weiner, L. M., Dhodapkar, M. V., and Ferrone, S. (2009). Monoclonal antibodies for cancer immunotherapy. Lancet 373, 1033-1040.

Weng, W. K., and Levy, R. (2003). Two immunoglobulin $\mathrm{G}$ fragment $\mathrm{C}$ receptor polymorphisms independently predict response to rituximab in patients with follicular lymphoma. J. Clin. Oncol. 21, 3940-3947.

Yamane, B. H., Hank, J. A., Albertini, M. R., and Sondel, P. M. (2009). The development of antibody-IL-2 based immunotherapy with hu14.18-IL2 (EMD-273063) in melanoma and neuroblastoma. Expert Opin. Investig. Drugs 18, 991-1000.

Yang, R. K., and Sondel, P. M. (2010). Anti-GD2 Strategy in the Treatment of Neuroblastoma. Drugs Future 35, 665.

Yu, A. L., Gilman, A. L., Ozkaynak, M. F., London, W. B., Kreissman, S. G., Chen, H. X., Smith, M., Anderson, B., Villablanca, J. G., Matthay, K. K., Shimada, H., Grupp, S. A., Seeger, R., Reynolds, C. P., Buxton, A., Reisfeld, R. A., Gillies, S. D., Cohn, S. L., Maris J. M., Sondel, P. M., and Group, C. S. O. (2010). Anti-GD2 antibody with GM-CSF, interleukin-2, and isotretinoin for neuroblastoma. N. Engl. J. Med. 363, 1324-1334. 
Zhang, W., Gordon, M., Schultheis, A. M., Yang, D. Y., Nagashima, F., Azuma, M., Chang, H. M., Borucka, E., Lurje, G., Sherrod, A. E., Iqbal, S., Groshen, S., and Lenz, H. J. (2007). FCGR2A and FCGR3A polymorphisms associated with clinical outcome of epidermal growth factor receptor expressing metastatic colorectal cancer patients treated with singleagent cetuximab. J. Clin. Oncol. 25, 3712-3718.
Zompi, S., and Colucci, F. (2005). Anatomy of a murder-signal transduction pathways leading to activation of natural killer cells. Immunol. Lett. 97, 31-39.

Conflict of Interest Statement: The authors declare that the research was conducted in the absence of any commercial or financial relationships that could be construed as a potential conflict of interest.
Received: 28 December 2011; accepted: 26 April 2012; published online: 16 May 2012.

Citation: Koehn TA, Trimble LL, Alderson KL, Erbe AK, McDowell KA, Grzywacz B, Hank JA and Sondel $P M$ (2012) Increasing the clinical efficacy of NK and antibody-mediated cancer immunotherapy: potential predictors of successful clinical outcome based on observations in high-risk neuroblastoma. Front. Pharmacol. 3:91. doi: 10.3389/fphar.2012.00091
This article was submitted to Frontiers in Pharmacology of Anti-Cancer Drugs, a specialty of Frontiers in Pharmacology.

Copyright (c) 2012 Koehn, Trimble, Alderson, Erbe, McDowell, Grzywacz, Hank and Sondel. This is an openaccess article distributed under the terms of the Creative Commons Attribution Non Commercial License, which permits non-commercial use, distribution, and reproduction in other forums, provided the original authors and source are credited. 